\title{
How and what others are doing in competitive intelligence: the Motorola case study
}

\author{
Marié-Luce Muller \\ IBIS Business and Information Services \\ mlm@ibis.co.sa \\ www.ibis.co.sa
}

\section{Introduction}

Recently, after a number of years of becoming increasingly involved in the execution of Competitive Intelligence (CI) as opposed to advising companies and organizations on CI structures, models and strategies, IBIS was approached by a large company to assist it in designing and implementing a CI capability. This was quite a novel assignment and once again highlighted the fact that seldom do two CI practices look alike. The reasons are multiple but perhaps the most logical reason is that no two companies or organization are the same. In nature they differ and also in size, the industry they play in, the manner in which they compete, their competitive strategies, the people and skills and many other reasons. Point is, there is no single model although there are common winning elements.

It would perhaps be useful to focus this column in this and in 2009 issues on examining various CI models, approaches and practices in a variety of companies differing in size, industry and structure, starting with one of the first companies that implemented CI, namely Motorola.

\section{About Motorola}

Motorola, Inc. is a publicly traded international communications company based in the USA, in a suburb of Chicago. The company began as the Galvin Manufacturing Corporation in 1928 by brothers Paul V. Galvin and Joseph E. Galvin. In 1930, Paul V. Galvin created the brand name Motorola for the company's new car radio. In 1947, Galvin Manufacturing Corporation changed its name to Motorola, Inc (Wikipedia 2009).

Motorola develops technologies, products and services, including communications infrastructure, enterprise mobility solutions, digital set-tops, cable modems, mobile devices and Bluetooth accessories. It is a Fortune 100 company with sales of US \$36.6 billion in 2007 (Motorola 2009). Motorola has the following main divisions:

1. Enterprise Mobility Solutions, headquartered in Illinois, USA, comprises communications offered to government and public safety sectors and enterprise mobility business.

2. Home and Networks Mobility, headquartered in Pennsylvania, USA, produces end-toend systems that facilitate uninterrupted access to digital entertainment, information and communications services via wired and wireless mediums.

3. Mobile Devices, headquartered in Illinois, USA and currently the least prosperous arm of the firm. It designs wireless handsets, but also licenses much of its intellectual properties. This includes cellular and wireless systems and as well as integrated applications, and Bluetooth accessories. 
In 2008, Motorola had 66000 employees (Wikipedia 2009).

\section{Motorola's CI development timeline}

Motorola's first encounter with CI was in 1983, even before the Society of Competitive Intelligence Professionals (SCIP) was founded in the US in 1986 (SCIP 2009). Motorola can be regarded as a leader in the development of CI in especially the early days of CI. The factor that got the company started was raised when its CEO Bob Galvin was appointed to the US President's Foreign Intelligence Advisory Board in the early 1980s. Experience and exposure there led to his conclusion that a CI department was essential for companies to gain competitiveness; he realized the value of focusing on the right things, collection and analysis. Galvin then approached a former CIA officer, Jan Herring to lead the corporate group (Herring 1999). This is an example of a proactive manner to get started in CI. Most companies however realize the need for CI only once they are in trouble or have been experiencing turbulent times or when they require key knowledge and then realize they do not have that knowledge or know how to create such knowledge. Still, today, it takes time before companies realize that the lasting benefits of CI requires CI to be a continuous process that is entrenched in the company culture. CI leaders advocate this need of viewing CI as a continuous full-time activity (Gilad and Gilad 1998).

The time that companies were hesitant to acknowledge that they practice CI is long forgotten. On the contrary, companies that do not engage in CI in one form or another are seen as not being competitive. Companies still call it different things but the two main terms are $\mathrm{CI}$ and Business Intelligence (BI). Motorola chairman and CEO Chris Galvin revealed how he specifically uses CI to maintain Motorola's competitive edge (Galvin 2000). He does not hide the fact that Motorola uses BI and he openly endorses it. This is one of the main success elements of $\mathrm{CI}$ - that the company leadership endorses, uses and drives the process and its outcome.

The figure below illustrates the development of CI in Motorola over time, depicting the activities in the green boxes and highlighting the challenges and shortcomings in the red boxes. Typical to a fledgling capability, in the experimental stages, CI was found to have been too theoretical and aimed to be the solution to all company needs (see Figure 1).

Figure 1 Experimental phase

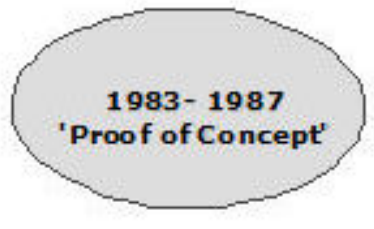

- Corporate mandate to establish struc ture for world-class u nit

- Strategy - top down

- Key Intelligence Topics (KIT) - direct interviews with top managers

- Service br oad needs of strategic and operational managers

- Professional intelligence collector s; library started

- Resour ces split to cover operational and strategic needs

- Too academic and reliant upon databases

Over time, the need for a centralized strategy became necessary along with the realisation that CI should ideally service the strategic needs of key clients. Motorola called this next developmental phase the credibility phase (Figure 2).

Figure 2 Building support 


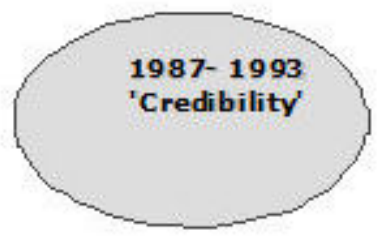

- Centralized strategy

- Rebuilt to focus on operational needs, business's relevance/development and ter hndogy

- 'The more people on the distribution list, the less value it has'

- Focus decreased internal Motorola networks and communication

- Lack of geographic coverage (as needed) - focused on 'mustdo's'

During the 1990s, CI in Motorola matured into a capability that had a solid internal network while its analysis capability became more globally focused to also include global strategic issues. The challenge then arose how to balance reactive and proactive activities (Figure 3).

Figure 3 CI maturing

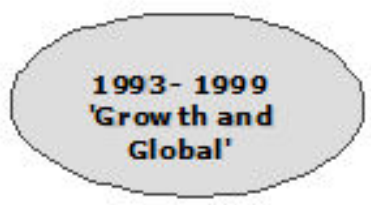

- Across and up strategy

- Build/br oaden global collection capabilities

- Redesign analysis to cover increasing global strategic issues

- Br oaden customers of intelligence: strategic focus + business specific

- Rebuildinternal intelligence network

- External intelligence netw orks decreased with re-focus on intemal sources as business is stressed

- Balance between reactive and proactive with additional coverage

At the turn of the century, Motorola identified a need to revisit its CI capability and return to basic principles to ensure that there is focus on the right issues, that collection continue and that analysis capabilities are adapted to include global issues relevant to Motorola. With a focus on company restructuring, the CI capabilities were, however, weakened, impacting negatively on the CI benefits (Figure 4).

Figure 4 Revisiting the aim

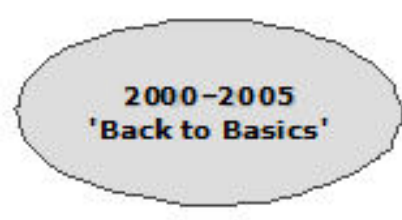

- Continue across and up strategy to customers of intelligence: strategic focus + business specific

- Global collection capabilities continue, but a estressed

- Redesign analytic products to cover increasing global strategic issues

- Re-str ucturing and business issues weakened intelligence team

- Dir ect contact with CEO and senior leadership star to weak, ends strong

CI in Motorola has experience upheavals over its 25 years of existence and has reached a mature phase in which it is enjoying the right support and control while delivering the right intelligence products. CI is also an integrated function in Motorola. The key challenge at present it to sustain the competitive culture in the company and retain the intelligence skills. Over the years the key intelligence needs or focus areas were adjusted as was necessary and 
at present the focus is mainly on honing and refining such as trade show intelligence collection and analysis, and ensuring quality input into the strategic planning processes in Motorola (Figure 5).

Figure 5 Growing again into maturity
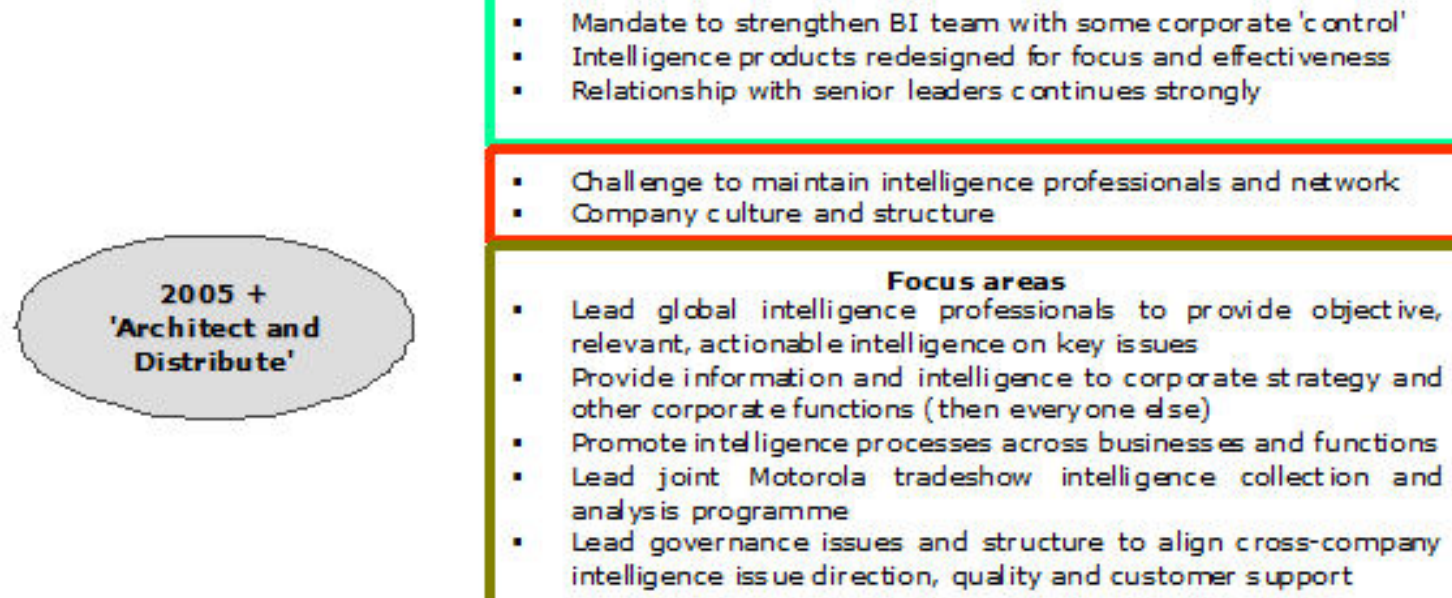

\section{Benefits of CI for Motorola}

As said repeatedly in the past, CI should never be a 'nice to have' and should bear real benefits for a company. Companies invest resources and expect return on that investment. Regarding Motorola, Galvin said in his opinion there are three main benefits of CI, namely early warning of opportunities and threats, objectivity of any competitive situation and an analysed input and insight into a competitive situation.

'With the exception of the surprise that comes through invention and innovation, we don't like to be surprised' (Chris Galvin, Motorola).

Motorola also regards CI's purpose to challenge the company's thinking around its market, its products, customers and competition, among others things (Prescott and Miller 2001). The company says it needs to get 'inside the head' of the competition and gain knowledge about how the competitors view their market and customers, technology and the future and, most importantly, provide insights into what models and plans the competitors have in approaching the future - how they react to change that is coming.

An important insight provided by Motorola's Galvin is that there are many areas of CI that could be outsourced to CI vendors but that, from a certain point, only its own analysts can develop unique insights and strategies in, for example, pricing and cost structures by assessing it from the company's vantage point, objectively and in depth.

'Next to knowing all about your own business, the best thing to know about is the other fellow's business' (John D. Rockefeller).

Galvin offered an example of how CI has paid off. In one case, the company learned that several rivals were interested in buying a company whose technology Motorola needed. As a result, Motorola bought a large stake in the targeted company, thereby thwarting any rival's ability to buy it. 


\section{Reporting structure for CI in Motorola}

In Motorola, corporate CI first reports to the chief strategy officer who in turn reports to the CEO and president (Goldberg 2008) (Figure 6). This is regarded as best practice in CI (Herring 2003 and 1999).

Figure 6 CI reporting structure in Motorola (Goldberg 2008)

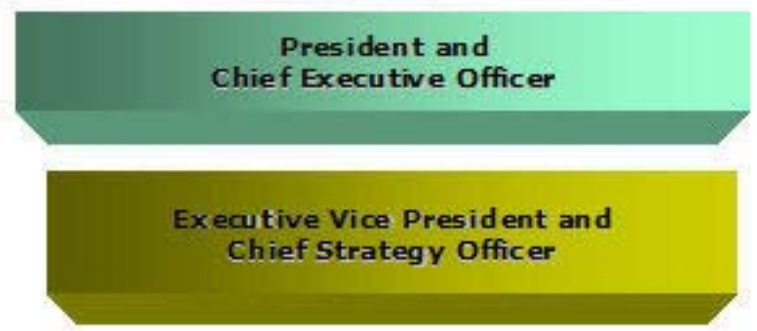

Corporate CI

\section{Hierarchy of internal clients of CI in Motorola}

Besides the reporting structure, there is a hierarchy of internal clients of CI in Motorola. The company distinguishes between primary and secondary clients but the main point is that CI supports a variety of clients company-wide (Figure 7). This has the benefit of ensuring participation and the maintenance of a competitive culture.

Figure 7 Primary and secondary clients of CI in Motorola (Goldberg 2008)

\section{Primary}

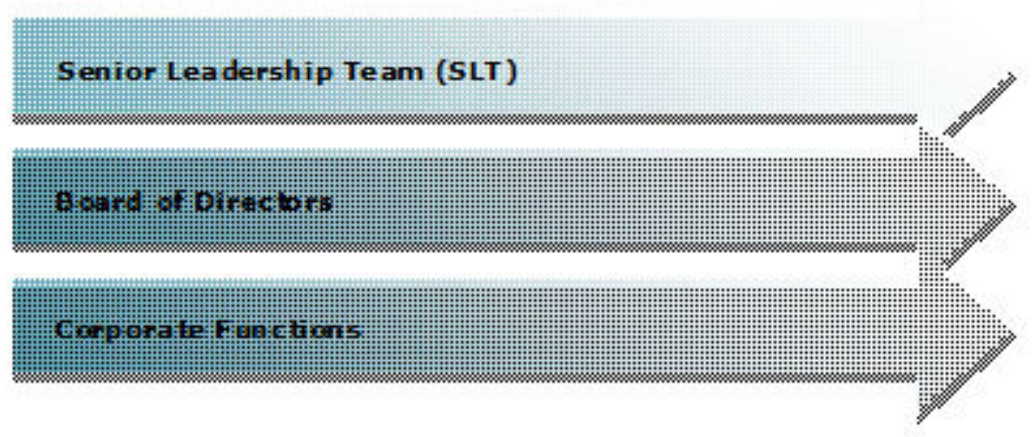

\section{Secondary}

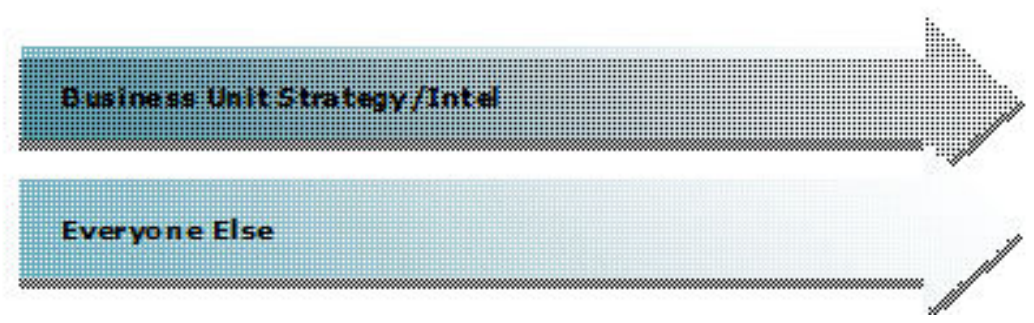

\section{Collection and input}


According to Goldberg (2008), CI historically ties together virtual teams in Motorola that ultimately report to the corporate $\mathrm{CI}$ function where data and information is captured in a centralized database, where after it can be evaluated, analysed, and interpreted (Figure 8).

Figure 8 Interaction among sources and networks in Motorola (Goldberg 2008)

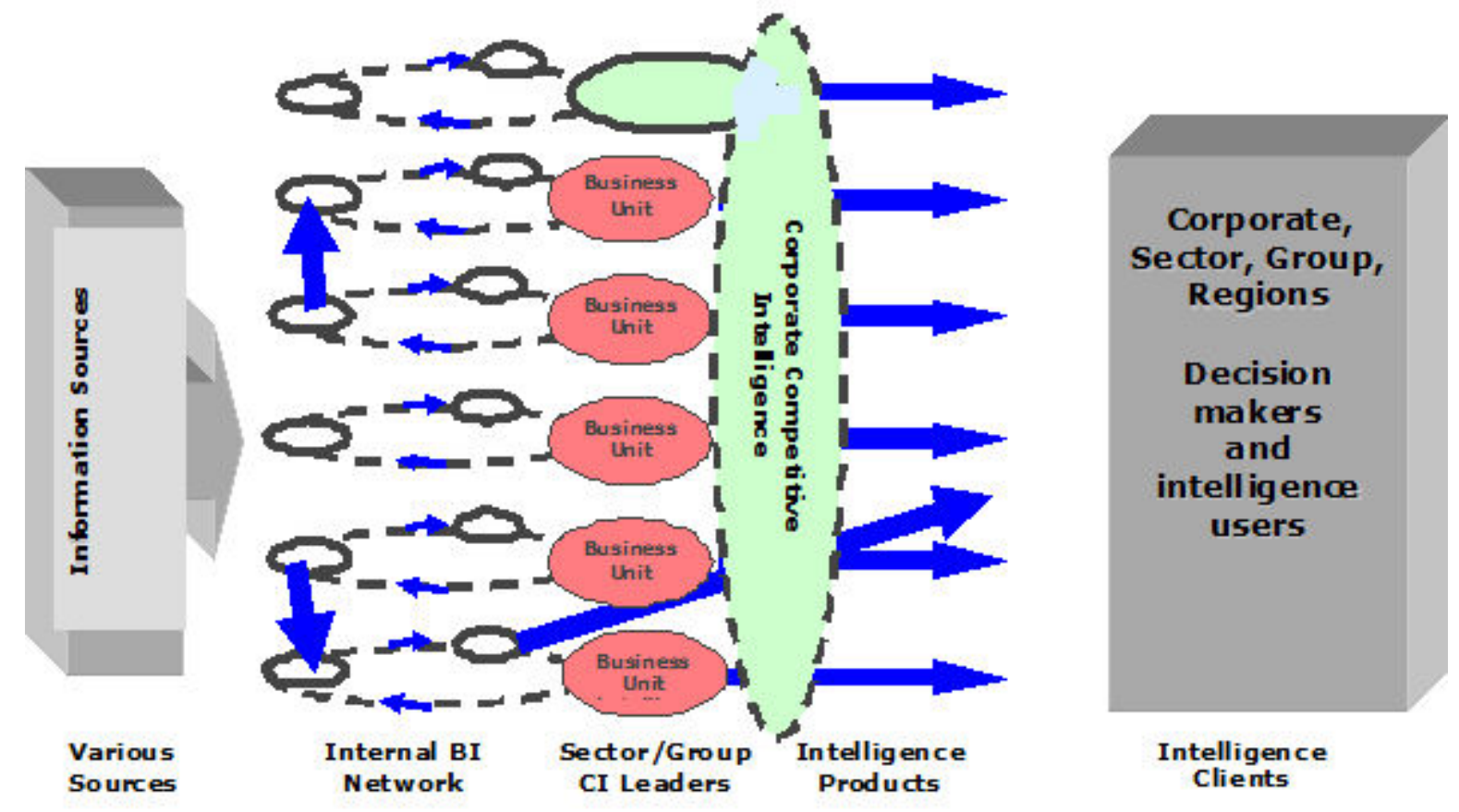

Key to proper CI is the ability to identify and access the right sources to collect the required data and information in a legal and ethical manner. Over the years and with the expansion of the Internet, the availability of sources has grown significantly. Key to good information, however, is the ability to utilize human sources and networks and to use the right techniques to gather information, be they Internet searches, personal interviews, studying analyst reports or observation techniques, among other things. Figure 9 shows the variety of sources that are used in Motorola.

Figure 9 Sources for Motorola (Goldberg 2008) 


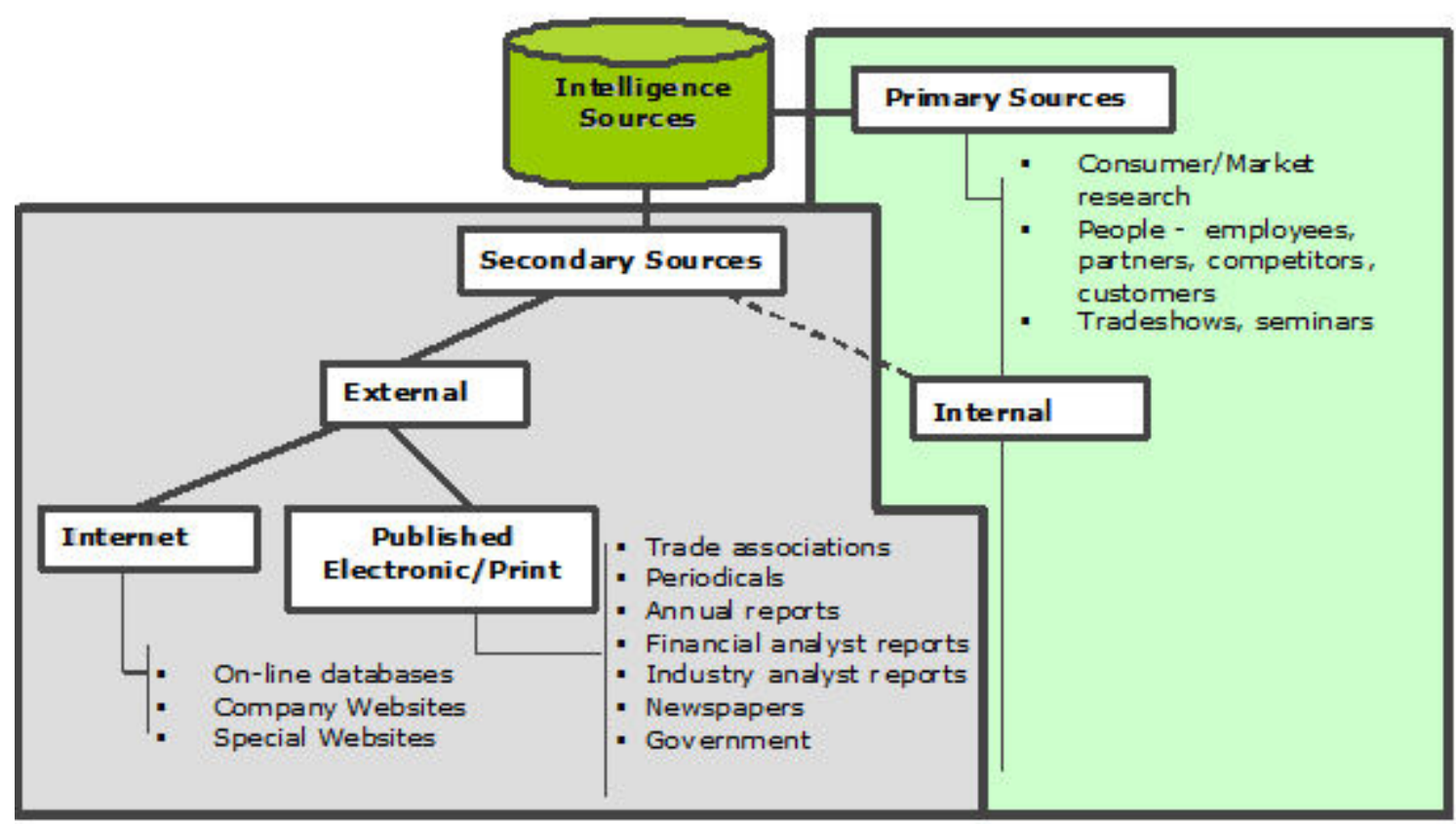

\section{Ingredients of effective CI in Motorola}

In Motorola, these ingredients are called critical elements that all have one purpose, namely to increase company revenue:

1. Management buy-in, support and drive to ensure that $\mathrm{CI}$ becomes and remains an integral part of strategy formulation. CI is first and foremost a strategic management instrument

2. Objectivity in analysis and the ability to design unique, company-specific insights that have a direct impact and/or relevance to business decisions

3. The ability to look further into the future and get a sense of where the market will be going, what the company can do now in proactive response and, even more importantly, what plans the competition is getting in place going into the future

4. Having the right people and skills is most important. Motorola's management mention a few key traits that need to be present in a CI professional in Motorola. These include an insatiable curiosity, critical thinking, a good retention capacity (memory), knowledge and instinct, information collection and analysis skills, people and communication skills, and the right training and education

5. Together with the forward-looking ability, CI should also be able to rapidly respond to market opportunities

6. Having a set of legal and ethical standards applies throughout the company at all times, including the process of gathering business intelligence so as to avoid potentially embarrassing and costly mistakes

7. Generally consistent corporate reporting structure and customers to maintain credibility, objectivity, relevancy and trust

8. CI should also alert to mistakes, security and defensive CI issues.

\section{Pitfalls that Motorola identified}

Motorola's CEO warns that there are pitfalls that can be avoided by being clear about what CI is and what it is not (Goldberg 2008). Key to understanding this aspect is to know that CI 
is the following:

1. A process to identify, collect and analyse key issues

2. Actionable analysis for key decision makers

3. Professional, efficient and effective

4. Endorsed by leadership

5. An objective perspective

6. Key in institutionalization, a CI culture company-wide.

The potential pitfalls include the notion that $\mathrm{CI}$ is an IT system able to produce the insights required. CI is also not the following (Goldberg 2008):

1. Primarily reactive research driven

2. A news, or data distribution service

3. An internally focused market research

4. A skill assumed to be inherent in all employees.

\section{Conclusion}

It is perhaps important to note that CI should be an integral part of a company in order to ensure it yields optimal benefits. It should further be noted that CI is not an insurance policy against all onslaughts, even though it might focus on the right things and be an effective strategic tool. It is, however, a strong and reliable fence against unsuspected and unwelcome surprises.

\section{References}

Galvin, C. 2000. Interview with Leonard Fuld on the Fuld Competitive Intelligence Center. [Online]. Available at www.factiva.com).

Goldberg, J. 2008. Competitive intelligence evolution at Motorola. [Online]. Available at http://blog.bloobble.com/2008/05/competitive-intelligence-do-you-know-what-motorola-isdoing/ (Accessed 21 February 2009).

Gilad, B. and Gilad, T. 1998. The business intelligence system. NY: American Management Association.

Herring, J, 1999. Key intelligence topics: a process to identify and define intelligence needs. Competitive Intelligence Review 10(2):4-14.

Herring, J. 2003. Identifying key intelligence topics. scip.online 35(July 14).

Motorola Website information, 2009. [Online]. Available at

http://mediacentrer.motorola.com. Accessed 21 February 2009.

Prescott, J.E. and Miller, S.H. (eds). 2001. Proven strategies in competitive intelligence lessons from the trenches. New York: Wiley.

Wikipedia. 2009. [Online]. Available at

http://wiki.howardforums.com/index.php/Motorola_Company_History. (Accessed 20

February 2009).

\section{About the author}

Marié-Luce Muller is a consulting CI analyst with IBIS Business and Information Services (Pty) Ltd, a leading Pretoria-based CI consultancy. She has a distinguished career in CI. Her primary experience lies in assisting companies in honing their CI capabilities. She also performs tracking and scanning activities on behalf of companies. Marié-Luce has published many articles on competitive intelligence (CEO Magazine, Finance Week, Business Week, Beeld, Die Burger and the South African Journal of Business Management), including an article on South Africa as an emerging CI player, which was published in an international publication of the Society of Competitive Intelligence Professionals (SCIP). She has also 
published a series of booklets on CI (Nuts and Bolts business series, published by Knowledge Resources) and is a member of a research team participating in an international study of CI practices among exporting companies. Previously, she was involved in research into the status of CI practices in South Africa. A member of SCIP, she holds a postgraduate degree from the University of Stellenbosch.

ISSN 1560-683X

Published by InterWord Communications for Department of Information and Knowledge Management, University of Johannesburg 